\title{
Co-autoria como indicador de redes de colaboração científica ${ }^{1}$
}

\author{
Maria de Fátima S. Maia
}

Mestre em comunicação e informação pela Universidade Federal do Rio Grande do Sul; Bibliotecária do Centro de Pesquisas Epidemiológicas da UFPel.

\section{Sônia Elisa Caregnato}

\section{Doutora em Comunicação e Informação pela Sheffield University; Professsora do Programa de Pós-Graduação em Comunicação e Informação da UFRGS.}

Estudo bibliométrico sobre redes de colaboração científica entre os professores do Programa de Pós-Graduação em Epidemiologia da Universidade Federal de Pelotas (PPGE/UFPel). As características de colaboração foram estudadas através das co-autorias dos artigos publicados em periódicos, no período entre 1991 e 2002. As análises revelaram que os professores publicam mais artigos em autoria compartilhada do que individual. Não foi encontrada relação entre o aumento da produtividade e um número maior de colaboradores, pois a taxa de produtividade e a taxa de autores por artigo não apresentam a mesma tendência; ou seja, o número de artigos publicados cresceu enquanto que o número de colaboradores permaneceu constante no período estudado. As análises de redes sociais revelaram uma configuração em torno dos professores mais produtivos.

Palavras-chave: Redes de colaboração científica; bibliometria; comunicação científica; redes sociais.

Extraído da dissertação de mestrado defendida no Programa de Pós-Graduação em Comunicação e Informação da Universidade Federal do Rio Grande do Sul, em julho de 2006, sob a orientação da professora Sônia Caregnato. Apoio financeiro: CAPES 


\section{Co-authorship as an indicator of scientific collaboration network}

The article is a bibliometric study on the scientific collaboration networks between professors in the Graduate Program in Epidemiology from the Federal University of Pelotas (PPGE/UFPel). The characteristics of the collaboration were studied by means of co-authorship of articles published in journals between 1991 and 2002. The analysis showed that professors publish more shared than single authorship articles. Correlation between productivity and collaboration was not found, since productivity rate and authorship rate per paper did not exhibit the same trend, i.e., the number of published papers increased whereas the number of collaborators was maintained. The analysis of social networks revealed a configuration around the most productive professors.

Keywords: Scientific collaboration networks; bibliometry; scientific communication; social networks.

Recebido em 02.01.2008 Aceito em 21.07.2008

\section{Introdução}

O homem sempre foi movido pela curiosidade e, neste sentido, a busca pelo conhecimento é tão remota quanto ele próprio. O conhecimento, seja ele empírico, moral, jurídico, religioso ou científico, não existe fora da relação do homem com a natureza e com outros indivíduos. A construção do conhecimento é um processo social realizado a partir do trabalho e do esforço coletivo e, sendo assim, é uma atividade humana movida pela curiosidade e construída socialmente (BOURDIEU, 2004; BURKE, 2003; MEADOWS, 1999; ZIMAN, 1979).

No âmbito da ciência, a imagem do pesquisador isolado faz parte do passado, pois atualmente o processo de produção científica requer associações, negociações e estratégias para interligar o maior número de elementos possíveis (SILVA, 2002). O compartilhamento de informações, a junção de competências e a união de esforços de pesquisadores na busca de metas em comum impulsionam a produção de conhecimento (BALANCIERI et al., 2005). O trabalho compartilhado proporciona economia de tempo e de recursos financeiros e materiais, e, portanto, é também estimulado pelas agências financiadoras de pesquisas. Todos esses fatores contribuem para que atualmente seja bastante valorizado o pesquisador capaz de formar boas, eficientes e produtivas equipes de 
trabalho. Além disso, o avanço das tecnologias de comunicação e as facilidades de deslocamento vêm proporcionando um aumento no número de estudos realizados de modo compartilhado e por meio de redes de colaborações, tanto entre indivíduos como entre instituições e países (CRONIN, 2005; GLANZEL, 2002; KRETSCHEMER, 2004; MEADOWS, 1999; SHIRABE e TOMIZAWA, 2002).

Paralelo ao aumento de estudos e publicações compartilhadas, cresce também o interesse em analisar essas colaborações, que podem apresentar diferentes características e variadas motivações. Independentemente das suas especificidades, é possível afirmar que a coautoria de produtos gerados pela atividade científica, particularmente de publicações, é um indicador de colaboração (CRONIN, 2005; KATZ e MARTIN, 1997; KIM, 2006; NEWMAN, 2004). Estudos sobre co-autoria têm sido empregados, por exemplo, para verificar diferenças entre colaborações acadêmicas e técnicas (MEYER e BHATTACHARYA, 2004), características de colaboração em determinadas disciplinas (GARG e PADHI, 2001; GLÄNZEL, 2002; NEWMAN, 2004; YOSHIKANE e KAGEURA, 2004), colaborações entre pesquisadores de mesmas ou diferentes instituições ou países (ARROYO-ALONSO, 2005; GLANZEL, 2001; KIM, 2006; MÄHLCK e PERSSON, 2000, SHIRABE e TOMIZAWA, 2002). Os resultados desses estudos revelam que as colaborações entre autores têm aumentado em todas as disciplinas. Entretanto, o grau de colaboração difere entre elas; ou seja, nas ciências naturais, por exemplo, não só o número de artigos em co-autoria como o número de autores por artigo é maior do que nas ciências sociais (MEADOWS, 1999; YOSHIKANE e KAGEURA, 2004; KATZ e MARTIN, 1997). Os estudos sobre co-autoria e colaboração mostram que geralmente trabalhos teóricos geram artigos com menos autores do que aqueles de natureza experimental (CRONIN, 2005; KATZ e MARTIN, 1997). Além disso, demonstram que os trabalhos cooperativos internacionais têm maior impacto e visibilidade (GLANZEL, 2002), e que a colaboração aumenta a produtividade dos pesquisadores (CRONIN, 2005; LEE e BOZEMAN, 2005).

Associado aos trabalhos sobre co-autoria, atualmente também se observa um fortalecimento do método de análise de redes sociais nos estudos sobre colaboração científica, o que possibilita uma visão abrangente das interações entre as partes, diferentemente das análises que enfocam características individuais. As análises de redes dão destaque para as relações, vínculos, influências e interações entre unidades, que podem representar tanto indivíduos quanto empresas, organizações, instituições ou nações. As redes sociais podem ser um conjunto de pessoas, instituições, ou organizações, que, por possuírem afinidades em comum, compartilham, por exemplo, trabalho e/ou informações e, por meio dessas ligações, vão construindo e re-construindo uma estrutura social (TOMAÉL e MARTELETO, 2005).

Tomando como referência trabalhos sobre co-autoria e análises de redes sociais, este artigo apresenta o resultado das análises sobre o processo de colaboração científica entre os professores do Programa de Pós-Graduação em Epidemiologia (PPGE) da Universidade Federal de 
Pelotas (UFPel), através das co-autorias nos artigos publicados em periódicos num período de doze anos. Os professores do PPGE da Universidade Federal de Pelotas, cidade localizada no extremo sul do estado do Rio Grande do Sul, são reconhecidos pelo trabalho com nível de excelência e desempenho equivalente aos dos mais importantes centros internacionais de ensino e pesquisa em sua área.

Os resultados destas análises buscam verificar se os professores do PPGE/UFPel são bastante colaborativos ou não, se essas colaborações aumentaram ou diminuíram ao longo do período estudado, se o número de colaborações proporciona um aumento da produtividade, se os professores colaboram mais com colegas ou com pesquisadores externos ao Programa, e se os professores mais produtivos também são mais colaborativos.

\section{Metodologia}

Para este estudo foram analisados os artigos publicados em periódicos pelos professores do PPGE/UFPel entre os anos 1991 e 2002. Como não é relevante apresentar os nomes dos professores, optou-se em utilizar códigos identificadores (P1 ...P12).

Maltrás Barba (2003) questiona as diferentes abordagens nas quantificações de colaboração científica. Devemos quantificar da mesma forma a colaboração entre um par ou entre um número maior de agentes? Pode-se considerar que a intensidade de colaboração é maior quanto menor for o número de participantes? Ou o inverso? Entretanto, também se pode considerar que a intensidade é a mesma em qualquer caso. 0 autor explica que cada uma das opções define uma diferente quantificação de colaboração: o cálculo denominado fracionado indica que o valor da colaboração diminui em proporção ao número de colaboradores, enquanto o cálculo denominado completo indica que a colaboração é maior quanto maior o número de colaboradores. Neste trabalho, primeiramente foi utilizado o método de quantificação denominado completo para verificar o número de autores por artigo e a sua relação com a produtividade dos professores. Depois, para suprir as carências resultantes do método de quantificação e complexificar os resultados obtidos, na busca de dados como a identificação de lideranças e grupos mais ou menos colaborativos, foi utilizado o método de análise de redes sociais.

A bibliografia foi organizada em um banco de dados no software EndNote $^{2}$, que permitiu importar registros bibliográficos de bases de dados como a PubMed ${ }^{3}$, facilitando significativamente a coleta de dados. Cada item foi devidamente analisado, identificando-se os nomes de todos os autores, ano de publicação, título dos periódicos e número de autores do artigo.

No que se refere ao controle de qualidade, todos os registros foram cuidadosamente conferidos, corrigindo-se, por exemplo, a grafia dos

\footnotetext{
${ }^{2}$ Disponivel em: <http://www.endnote.com>.

${ }^{3}$ Disponivel em: <http://www.pubmed.gov>
} 
nomes de autores e evitando assim duplicações. Após esse processo, os registros foram transferidos para os aplicativos Microsoft Excel e SPSS ${ }^{4}$, que permitiram analisar e representar graficamente as características de colaboração entre os professores. As análises de redes sociais foram feitas através do software UCINET ${ }^{5}$.

Nas redes sociais, um dos aspectos importantes a serem analisados é a posição que os atores ocupam em relação a toda a estrutura da rede, e, dentro desse contexto, se destaca a posição de centralidade. A posição de centralidade é associada ao poder, ou seja, estar no centro da rede significa ter menos restrições e mais oportunidades através das relações que se estabelecem entre os atores. Hanneman (1998) enfatiza que definir uma posição mais ou menos favorável não é uma tarefa simples e definitiva, mas as análises de redes sociais têm contribuído bastante para uma melhor compreensão do poder que pode ser atribuído a determinados atores dentro das estruturas de relações sociais. Marteleto afirma que "calcular a centralidade de um ator significa identificar a posição em que ele se encontra em relação às trocas e à comunicação na rede" e acrescenta ainda que "[...] quanto mais central é um indivíduo, mais bem posicionado ele está em relação às trocas e à comunicação, o que aumenta seu poder na rede" (MARTELETO, 2001, p.76). A Figura 1 apresenta três exemplos que representam diferentes posições dos atores de uma rede.
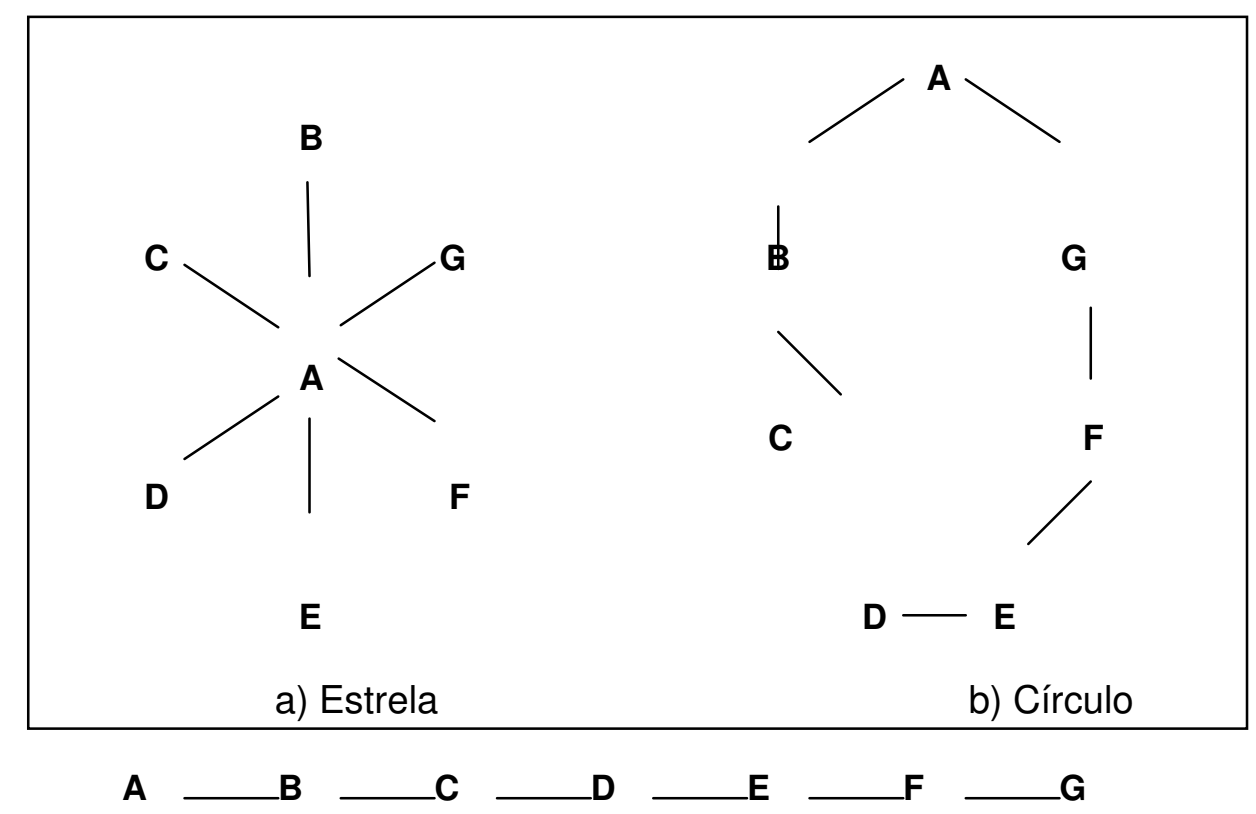

c) Linha

Figura 1 - Três tipos de centralidade nos estudos de redes sociais. FONTE: WASSERMAN, S.; FAUST, K., 1999, p. 171.

\footnotetext{
${ }^{4}$ Disponível em: <http://www.spss.com.br $>$.

${ }^{5}$ Disponível em: <http://www.analytictech.com $>$.
} 
Na rede Estrela da Figura 1, o ator A ocupa uma posição de vantagem em relação aos demais, pois, se o ator $C$, por exemplo, privar $A$ de alguma informação ou recurso, A poderia recorrer a outras cinco possibilidades de intercâmbio, enquanto $D$ não teria mais nenhuma. Nas análises de redes sociais se avalia que o ator $A$ tem um grau seis de centralidade, enquanto os demais têm grau um, e, quanto maior for o grau de centralidade de um ator, maior será seu poder dentro da rede. No exemplo da rede Círculo, todos os atores têm o mesmo número de conexões ou vínculos e, sendo assim, ocupam a mesma posição e apresentam o mesmo grau de centralidade e poder. Na rede Linha os atores $A$ e $G$ estão em desvantagem e os demais têm o mesmo grau de centralidade (HANNEMAN, 1998; WASSERMAN e FAUST, 1999).

Além do grau de centralidade, nas análises de redes sociais também se pode avaliar o grau de proximidade de determinado ator. Na rede Estrela, por exemplo, o ator A apresenta uma posição mais favorável quanto ao grau de proximidade, pois tem possibilidade de contato direto com todos os outros atores da rede. Aqueles que apresentam menores distâncias em relação aos demais apresentam um grau maior de proximidade, e, estando mais próximos dos demais, são mais acessíveis, o que significa uma posição favorável e vantajosa. Na rede de Círculo todos os atores apresentam distribuições idênticas quanto à proximidade e, portanto, ocupam posições iguais na estrutura. Na rede Linha, novamente os atores A e $G$ estão em desvantagem, pois estão mais próximos apenas de $B$ e $F$ respectivamente, enquanto os demais estão mais próximos de um número maior de atores e, sendo assim, ocupam posições mais favoráveis (HANNEMAN, 1998).

Além da centralidade e da proximidade, nas análises de redes sociais também é utilizado o grau de intermediação, que significa a posição que determinado ator ocupa em relação a um par de atores. Novamente, o ator A da rede Estrela (Figura 1) apresenta vantagem, pois está posicionado entre todos os outros pares de atores da rede. Ou seja, para que haja contato entre $B$ e $E$ ou $C$ e $F$, por exemplo, é necessário primeiro contato com A. Quanto mais atores dependerem de A para realizar conexões, mais poder ele tem de negociar esses contatos e, se for o caso, estimular ou não essas conexões. O grau de intermediação é calculado a partir da freqüência com que cada ator aparece nos caminhos, entre todos os pares de atores da rede (HANNEMAN, 1998).

\section{Resultados}

No período compreendido entre 1991 e 2002, doze professores atuaram no Programa de Pós-Graduação em Epidemiologia da Universidade Federal de Pelotas, publicaram 237 artigos em 81 diferentes periódicos (28 do Brasil e 53 do exterior), envolvendo 369 autores, incluindo autores e co-autores. 
Verificou-se que a maioria dos artigos foi de autoria múltipla. Nos 237 artigos analisados, somente 17 (7\%) tiveram autoria individual, sendo que os 220 restantes (93\%) apresentaram dois ou mais autores, sendo que o artigo com maior número possui 20 autores. $O$ fato de serem poucos os artigos com autoria individual já demonstra, por si só, uma característica essencialmente colaborativa entre a equipe.

A FIG. 2 apresenta a relação entre a distribuição de artigos segundo o número de autores, na qual se pode concluir que a maioria dos artigos dos professores do PPGE/UFPel apresentou cinco, quatro ou três autores.

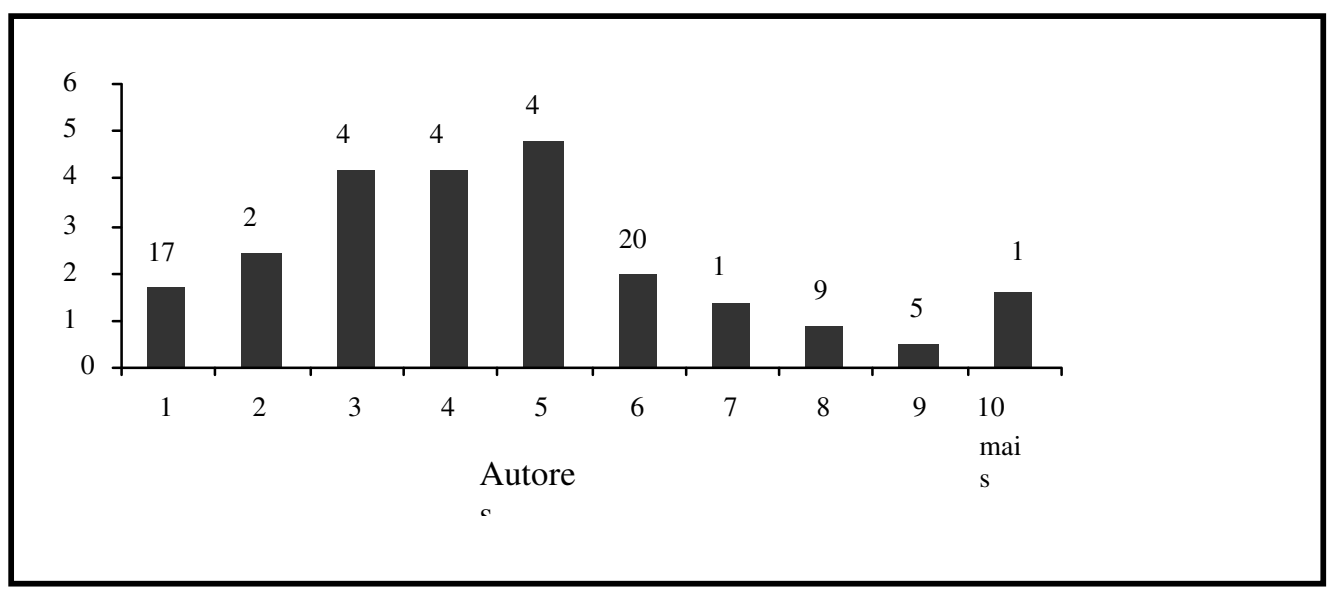

Figura 2 - Distribuição dos artigos de acordo com o número de autores (PPGE/UFPel - 1991 - 2002).

FONTE: Dados da pesquisa.

Meadows (1999) afirma que os pesquisadores mais produtivos tendem a ser mais colaborativos. Esta afirmação se confirma neste estudo, pois, tomando como exemplo o autor mais produtivo (P4 com 139 artigos publicados no período, média de um artigo por mês), a maior parte da sua produção apresenta autoria coletiva (95\%). Sendo assim, pode-se afirmar que o autor mais produtivo é predominantemente colaborativo.

Analisando a totalidade da produção científica destes autores, não houve relação entre produtividade e colaboração, pois a taxa de produtividade e a taxa autores por artigo não apresentaram a mesma tendência, ou seja, o número de artigos publicados cresceu enquanto o de colaboradores permaneceu constante. Sendo assim, as parcerias foram constantes e estáveis no que se refere ao número de autores por artigo.

Glänzel e Schubert (2004) apontam que há padrões específicos de relação entre produtividade e colaboração para diferentes campos científicos, isto é, a colaboração promove o aumento da produtividade até atingir um limite, e depois este nível de correlação se torna negativo. Eles acrescentam ainda que estes limites variam de acordo com as particularidades das disciplinas, de 1-2 co-autores em matemática, acima 
de 3-4 em química, e 5-6 co-autores nas áreas biomédicas. Este mesmo padrão também foi observado neste estudo, conforme mostra a FIG. 2, abaixo. A observação coincidente desta pesquisa com a de Glänzel e Schubert (2004) reforça esta tendência de comportamento colaborativo nas áreas biomédicas.

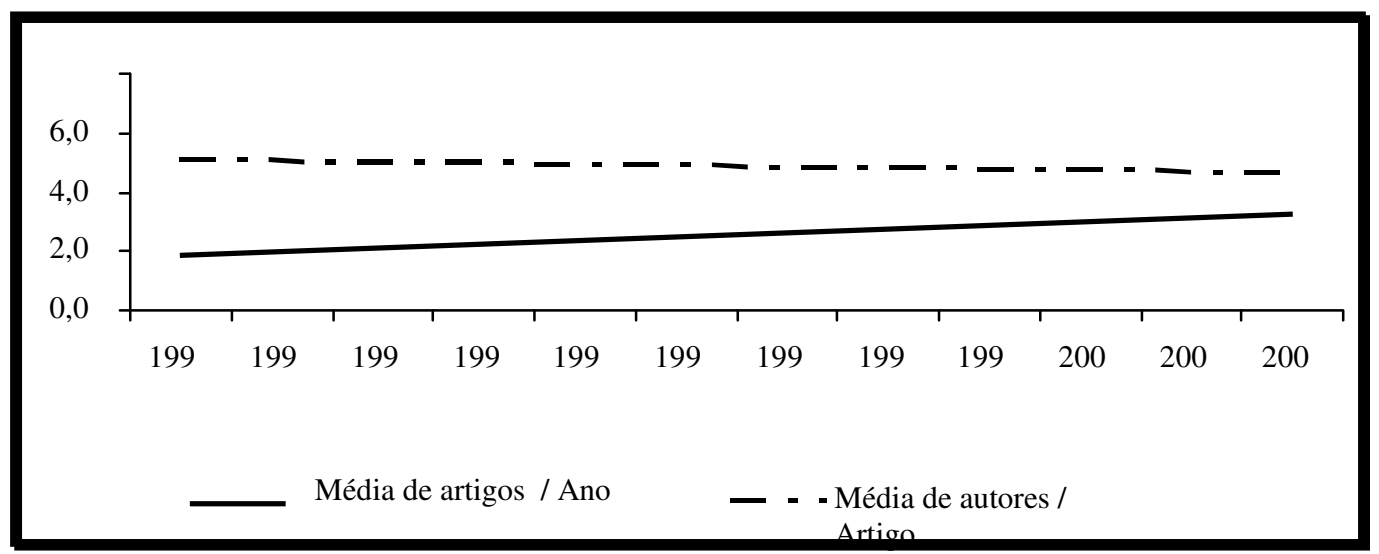

Figura 3 - Tendência da produtividade e média de autores por artigo (PPGE/UFPel 1991- 2002).

FONTE: Dados da pesquisa.

Nos estudos sobre colaboração científica, alguns autores apontam que calcular o coeficiente de colaboração (collaborative coeficiente - CC) é o método mais sensível de se avaliar se há crescimento do número de autores por documento e também de trabalhos com múltipla autoria (GARG e PADHI, 2001). O coeficiente de colaboração é uma medida proposta por Ajiferuke e colaboradores (1988 apud GARG e PADHI, 2001), a partir da idéia de produtividade fracionada proposta por Price e Beaver $^{7}$ (1966 apud GARG e PADHI, 2001). O cálculo do coeficiente de colaboração (CC) é feito através da seguinte equação:

$C C=1-\left\{\sum_{j=1}^{n}(1 / j) F j / N\right\}$, sendo que:

$F j=$ número de documentos com $j$ autores durante um período de tempo determinado.

$N=$ total de documentos publicados durante um período de tempo determinado

\footnotetext{
${ }^{6}$ AJIFERUKE, I; BURREL, Q; TAGUE, J. Collaborative coefficient: a single measure of the degree of collaboration in research. Scientometrics, v. 14, p. 421-33, 1988.

7 PRICE, D. S.; BEAVER, D. B. Collaboration in an invisible college. American Psychologist, v. 21, p. 1011, 1966.
} 
Aqui se aplicou a equação para cada ano separadamente (de 1991 até 2002). Na Figura 6 pode-se verificar os coeficientes de colaboração de cada ano, assim como a tendência de todo o período. O resultado do cálculo do coeficiente de colaboração (CC) será sempre um número entre 0 e 1 , no qual um valor mais próximo de 1 significa mais colaboração (GARG e PADHI, 2001).

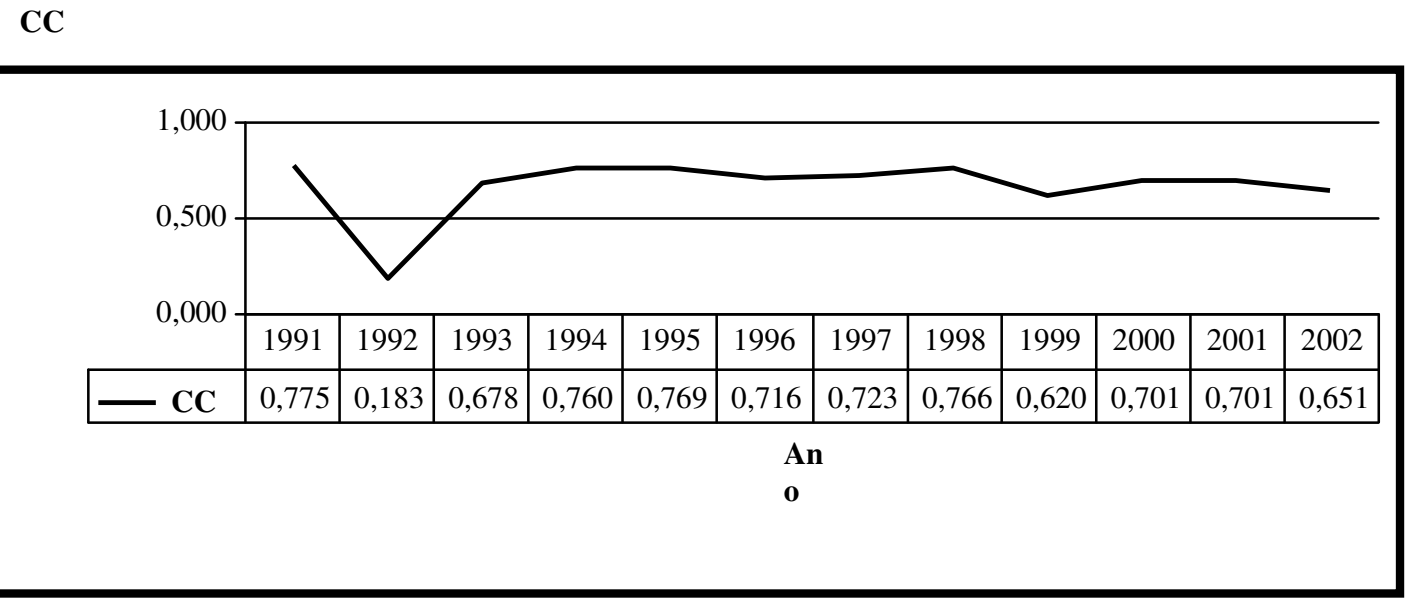

Figura 4 - Coeficientes de colaboração dos professores do PPGE/UFPel (1991 - 2002).

FONTE: Dados da pesquisa.

Na Figura acima se pode observar que o coeficiente de colaboração entre os professores do PPGE/UFPel é alto, exceto no ano de 1992 em que houve uma queda. O restante do período apresentou um padrão praticamente constante. Pode-se concluir que a quantidade de autores envolvidos no processo de produção de artigos não proporcionou o seu aumento.

Ainda optou-se em conferir com quem cada professor partilhou um maior número de trabalhos. Verificou-se que todos os professores tiveram um número maior de colaborações com os colegas do Programa, exceto P6 e P9, que colaboraram mais com autores externos ao PPGE/UFPel. Cinco dos 12 professores tiveram um número maior de trabalhos compartilhados com P4 (que também é o professor com maior número de publicações). Também foram identificados dois pares de professores que colaboram bastante entre si: P2 com P10 e P4 com P7.

Por fim, para melhor conhecer as colaborações entre os professores do PPGE/UFPel, verificamos esse processo através das análises de redes sociais, o que veremos a seguir.

O primeiro passo constituiu-se em construir uma tabela que apresenta o número de colaborações de cada membro da equipe com seus respectivos colegas.

Vale ressaltar que a rede de atores aqui observados é construída a partir das co-autorias dos artigos publicados pelos professores do PPGE/UFPel. Sendo assim, não há como um professor ser co-autor de um 
colega sem que o inverso não aconteça, esse tipo de relação caracteriza o que se chama de rede simétrica. Em outras palavras, P2 é colaborador de P10 o mesmo número de vezes que P10 é colaborador de P2. Existem as redes assimétricas nas quais as relações de $\mathrm{P} 2$ com $\mathrm{P} 10$ poderiam ser diferentes das de P10 com P2, entretanto esse não é o caso quando se trabalha com co-autorias.

$\mathrm{Na}$ TAB. 2 também se podem examinar dois diferentes dados: 0 número de colaborações e a quantidade de diferentes atores com quem se deram as colaborações. Um professor pode ter 10 colaborações com um único colega, ou pode ter 10 colaborações com cinco diferentes colegas (duas com cada). A Figura 4 é o grafo que representa as colaborações entre os professores do PPGE / UFPel no período entre 1991 e 2002.

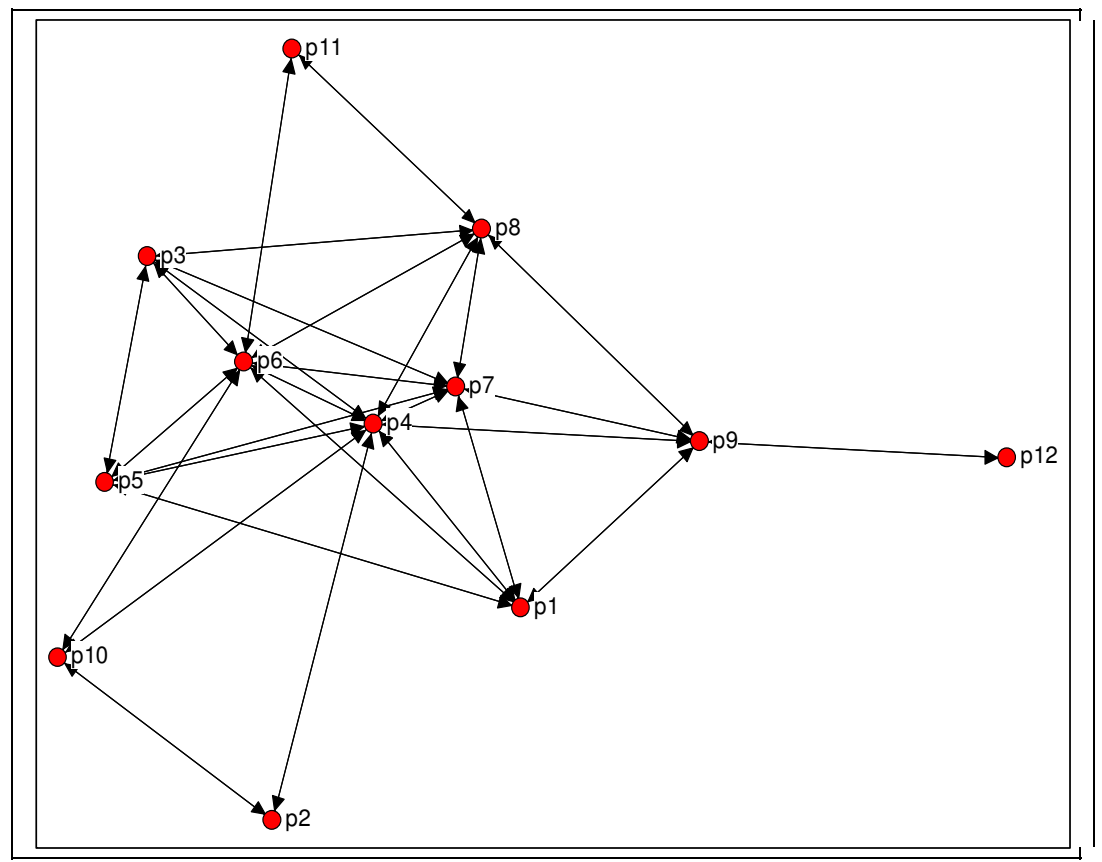

Figura 5 - Representação das colaborações entre os professores do PPGE/UFPel (1991-2002).

FONTE: Dados da pesquisa.

Ao observar a Figura 4 se verifica que há uma grande quantidade de linhas relacionais entre os atores da rede, significando uma rede densa no que diz respeito à produção de artigos de periódicos. Pode-se afirmar também que, no período analisado, houve bastante comunicação entre os professores do PPGE/UFPel, o que proporciona um número grande de artigos compartilhados.

Alguns professores não colaboraram diretamente, mas se observa que a distância entre os mais afastados é pequena (três graus no 
máximo). Por exemplo, se P2 quisesse colaborar com P12 bastaria contatar com P4 e P9.

O professor P4 é o que obteve um número maior de conexões com uma quantidade maior de colegas, mesmo que não tenha colaborado diretamente com P11 e P12. A rede aqui analisada se configura ao redor dos atores mais produtivos, isto é, aqueles que apresentaram as maiores médias de artigos por ano (P4, P7 e P6).

Os professores P2, P11 e P12 ocupam posições periféricas em relação aos colegas de equipe, mas vale salientar que essa característica não significa serem pesquisadores que trabalhem totalmente isolados, pois, provavelmente, fazem parte de outras redes que não estão aqui representadas. Os atores mais periféricos eventualmente representam a ligação ou a ponte entre a equipe do PPGE/UFPel e pesquisadores externos ao Programa. Pode-se verificar, por exemplo, que P10 ocupa uma posição periférica na rede, mas ao analisar a Tabela 1 percebe-se que apresentou uma média maior de autores por artigo, sendo assim, os autores com quem ele mais colaborou são externos ao PPGE/UFPel. As colaborações são motivadas, principalmente, por interesses em comum entre os atores, e, dependendo das linhas de pesquisa de cada um, investigações e artigos são compartilhados ou não.

Para uma melhor interpretação dos grafos é possível, através do software Ucinet, calcular o grau de centralidade (centrality), intermediação (betweenness) e proximidade (closeness) para cada professor; essas medidas reforçam o que o grafo é capaz de demonstrar e auxiliam no seu entendimento. Entretanto, vale ressaltar que essas posições centrais ou periféricas não são características particulares dos professores, são sempre em função das relações com os outros atores da rede. É bom lembrar que as análises de redes sociais se referem às relações entre os atores e, sendo assim, apresentam características relacionais.

O cálculo do grau de centralidade mostrou destaque para P4, P6 e P7, que ocupam a parte central da Figura e, portanto, possuem mais relações de troca e comunicação, o que aumenta seu poder na rede. Quanto à característica de proximidade, que significa a capacidade que um determinado ator tem para chegar aos demais, se destacaram os professores P4, P7 e P6. Em relação ao grau de intermediação, que é a capacidade que determinado ator tem de intermediar uma relação entre outros pares, as análises demonstraram um grau maior para P4, seguido de P9 e P6.

Conclui-se então que P4 e P6 (que tiveram destaque nas três medidas) são os professores com maior capacidade de intermediar as relações, que estão mais próximos dos demais atores da rede e, portanto, ocupam posições de liderança junto aos demais professores do PPGE/UFPel. O professor P7 apresentou percentuais de centralidade e proximidade altos, mas não se destacou na característica de intermediação. 


\section{Conclusão}

No que diz respeito às colaborações, as análises mostraram que os professores do PPGE/UFPel publicaram mais artigos de autoria compartilhada. Não foi encontrada relação entre 0 aumento da produtividade com um número maior de colaboradores, pois a taxa de autores por artigo não apresentou a mesma tendência da produtividade, isto é, o número de artigos cresceu no período, enquanto o número de colaboradores foi mais constante. Verificou-se que as colaborações se repetiram, significando que a equipe foi consolidando as relações e aumentando a produtividade. Os principais colaboradores dos professores foram colegas da mesma equipe.

As análises de redes sociais revelaram uma configuração em torno dos professores mais produtivos. Os professores P4, P6 e P7, que publicaram um maior número de artigos, também apresentaram um maior número de linhas relacionais na rede e, portanto, ocupam posições de liderança junto aos demais e são capazes de influenciar o trabalho de todos.

Em síntese, a produtividade aumentou, ao passo que as colaborações ficaram estáveis, apresentando, inclusive, nos últimos anos, uma leve queda na taxa média de colaboradores por artigo. Agora resta verificar a visibilidade do trabalho dos professores, pois, a partir do impacto dos artigos na literatura nacional e internacional, será possível delinear se as estratégias de produção e publicação utilizadas estão dando bons resultados ou se precisam ser alteradas.

Futuramente também será válido investigar em maior profundidade a característica endógena encontrada entre os professores do PPGE/UFPel, assim como as redes de colaboração com pesquisadores externos ao Programa. Isso permitirá esclarecer se neste caso também a co-autoria internacional resulta em publicações com maior fator de impacto do que a co-autoria doméstica, como constataram Glänzel e Schubert (2001).

\section{Referências}

ARROYO-ALONSO, A. Estudio cienciométrico de la colaboración científica en la Universidad Politécnica de Valencia, Espanha. Information Research, Lund, v.11, n.1, 2005. Disponível em: <http://InformationR.net/ir/111/paper245.html>. Acesso em: 10 mar. 2006.

JIFERUKE, I; BURREL, Q; TAGUE, J. Collaborative coefficient: a single measure of the degree of collaboration in research. Scientometrics, v. 14, p. 421-33, 1988 citado por GARG, K. C.; PADHI, P. A study of collaboration in laser science and technology. Scientometrics, Amsterdam, v.51, n.2, p.415-27, 2001.

BALANCIERI, R. et al. A análise de redes de colaboração científica sob as novas tecnologias de informação e comunicação: um estudo na Plataforma Lattes. Ciência da Informação, Brasília, v.34, n.1, p.64-77, 2005. 
BOURDIEU, P. Os usos sociais da ciência: por uma sociologia clínica do campo científico. São Paulo: UNESP, 2004. 86 p.

BURKE, P. Uma história social do conhecimento: de Gutenberg a Diderot. Rio de Janeiro: Jorge Zahar, 2003.

CRONIN, B. The hand of science: academic writing and rewards. Oxford: Scarecrow Press, 2005. 214 p.

GARG, K. C.; PADHI, P. A study of collaboration in laser science and technology. Scientometrics, Amsterdam, v.51, n.2, p.415-27, 2001.

GLÄNZEL, W. National characteristics in international scientific coauthorship relations. Scientometrics, Amsterdam, v.51, n.1, p.69-115, 2001.

. Coauthorship patterns and trends in the sciences (1980-1998): a bibliometric study with implications for database indexing and search strategies. Library Trends, Urbana, v.50, n.3, p.461-73, 2002.

GLÄNZEL, W.; SCHUBERT, A. Double effort = Double impact? A critical view at international co-authorship in chemistry Scientometrics, Amsterdam, v.50, n.2, p.199-214. 2001.

. Analysing scientific networks through co-authorship. In: MOED, $\mathrm{H}$. F. Handbook of quantitative science and technology research: Dordrecht, Netherlands: Kluwer Academic Publishers, 2004. p. 257-276

HANNEMAN, R. A. Introduccion a los métodos del analisis de redes sociales. Riverside: Universidad de California Riverside, 1998. Disponível em: <http://revista-redes.rediris.es>. Acesso em: 10 maio 2006.

KATZ, J. S.; MARTIN, B. R. What is research collaboration? Research Policy, London, v.26, p.1-18, 1997.

KIM, K.-W. Measuring international research collaboration of peripheral countries: taking the context into consideration. Scientometrics, Amsterdam, v.66, n.2, p.231-40, 2006.

KRETSCHMER, $H$. Author productivity and geodesic distance in bibliographic co-authorship networks, and visibility on the Web. Scientometrics, Amsterdam, v.60, n.3, p.409-20, 2004.

LEE, S.; BOZEMAN, B. The impact of research collaboration on scientific productivity. Social Studies of Science, New York, v.35, n.5, p.673-702, 2005.

MÄHLCK, P.; PERSSON, O. Socio-bibliometric mapping of intradepartmental networks. Scientometrics, Amsterdam, v.49, n.1, p.81-91, 2000.

MALTRÁS-BARBA, B. Los indicadores bibliométricos: fundamentos y aplicación al análisis de la ciencia. Gijon: Trea, 2003. 
MARTELETO, R. M. Análise de redes sociais: aplicação nos estudos de transferência da informação. Ciência da Informação, Brasília, v.30, n.1, p.71-81, 2001.

MEADOWS, A. J. A comunicação científica. Brasília: Briquet de Lemos, 1999. $268 \mathrm{p}$.

MEYER, M.; BATTACHARYA, S. Commonalities and differences between scholary and technical collaboration: an exploration of co-invention and co-authorship analyses. Scientometrics, Amsterdam, 61, n.3, p.443-56, 2004.

NEWMAN, M. E. J. Coautorship networks and patterns of scientific collaboration. PNAS, Washington, v.101, Suppl. 1, p.5200-05, 2004.

SHIRABE, M.; TOMIZAWA, $H$. Likelihood of overseas access to international co-autorship. Scientometrics, Amsterdam, v.53, n.1, p.123$29,2002$.

SILVA, E. L. Rede científica e a construção do conhecimento. Informação e Sociedade: Estudos, João Pessoa, v.12, n.1, p.120-48, 2002.

TOMAÉL, M. I.; MARTELETO, R. M. Redes sociais: posições dos atores no fluxo da informação. Enc. Bibli: R. Eletr. Bibliotecon. Ci. Inf., Florianópolis, n. esp., 2006.

WASSERMAN, S.; FAUST, K. Social network analysis: methods and applications. Cambridge: Cambridge University Press, 1999. 825 p.

YOSHIKANE, F.; KAGEURA, K. Comparative analysis of coauthorship networks of different domains: the growth and changes networks. Scientometrics, Amsterdam, v.60, n.3, p.433-44, 2004.

ZIMAN, J. M. Conhecimento público. Belo Horizonte: Itatiaia, 1979. 